\title{
Challenges in thermal management for electrified vehicles
}

Heike Schönerstedt

Daimler AG

This manuscript is not available according to publishing restriction. Thank you for your understanding. 